\title{
En torno a los Diurnali del Duca di Montelione
}

\author{
Avelino Sotelo Álvarez
}

\section{Introducción}

Los Diurnali del Duca di Montelione reciben tal nombre por haber sido Ettore Pignatelli, primer duque de Montelione, el poseedor del códice manuscrito. Dicho duque murió en 1535, cuando era virrey de Sicilia de la monarquía católica.

El manuscrito narra la historia del reino de Nápoles desde 1266, en que reina Carlos de Anjou, "Rex utriusque Siciliae», hasta 1478, que comprende el período de la conquista de Nápoles por Alfonso V de Aragón, con Nápoles capital del reino y la instauración de su hijo bastardo Ferrante I de Aragón.

El autor del manuscrito Montelione ${ }^{1}$ es incierto, aplane incertus", dice Muratori en el Praefatio a su compilación-rifacimento; el manuscrito llevaba inscrito solamente el título Li Diurnali del Duca di Montelione. Sirvió de fuente histórica en el siglo XVI a Angelo di Costanzo para su Historia d'incerto autore ${ }^{2}$ y a G. Summonte en $1601^{3}$. Asimismo, Zurita en sus Anales de la Corona de Aragobn $n^{4}$ hace referencia y transcribe párrafos completos del que llama uautor antiguo", aunque tanto éstos como Buonincontro y los cronistas e historia-

1. De ahora en adelante con la abreviación DDM.

2. di Costanzo, A. Dell Tstoria del Regno di Napoli dincerto Autore, la quale comincia dalla morte di Carlo li d'Angiò e termina col Regno di Alfonso I d'Aragona. Napoli, Nella stamperia di G. Gravier, 1769 , con lizenza dei Superiori.

Leemos en el prólogo: «L'Editore ai benigni lettori... Da tali parole ed espressioni pareche possa affermarsi di sicuro che l'Autore scrisse questa Istoria nel Pontificato di Paolo IV, che durò dal 1555 al 1559, prima che verisse la Storia del Costanzo, i primi orto libri della quale non furono pubblicati che nel $1572 \ldots$ e gli aleri libri nel $1582 \ldots$... Nell'uno e nell altro Autore molti pasi simili ed expresioni quasi co' medesimi sentimenti e parole. Cosi è facile di osservare in que'luoghi dove tutti c due Autori parlano della regina Giovanna I e di Carlo III di Durazzo e dell'ingrandimento dei fratelli della famosa Lucretia d'Alagno... L'Autore śappella Innocenzo Fuidoro.... alla p. $217 \ldots$, que hoy la crítica unánimemente asigna al mismo A. di Costanzo.

3. Summonte, G. Historia de la Città e Regno di Napoli. Napoli, Gio. Jacomo Garlenio, 1601.

4. Zurita, G. Anales de la Corona de Aragón. Zaragoza, H, nos P. Lanaja y Lamarca, 1669. A costa del mismo Reyno. 
dores, como Scipione Ammirato, Costo, Giovio y más tarde Muratori en el siglo XVIII, se sirvieron de las varias copias que se hicieron desde fines del siglo XV y todo el XVI.

El manuscrito más antiguo se supone que es de fines del siglo XV o principios del siglo XVI. Este fue publicado a fines del siglo XIX entre los monumentos históricos de la Società Napolitana di Storia Patria, tras haber sido adquirido por dicha Società, en la cual F.N. Faraglia ${ }^{5}$ fue el que cuidó la publicación, enriqueciéndola con variantes de otros dos manuscritos, uno de la Biblioteca de San Martino de Nápoles, adquirido por la Biblioteca Nacional de la misma ciudad, y otro de la misma Biblioteca Nacional, que tenía este título: aLibro aulico, trovato in casa del Duca di Montelione, nel quale sono annotate le cose successive dall'anno 1262 all'anno 1457 n, que procede de los libros de Pietro Pollidoro ${ }^{6}$.

Otros manuscritos proceden de copias retocadas y con añadiduras, hechas en el siglo XV1. A veces estos urifacimentin cambian la misma personalidad lingüística y estilística del autor original por la abreviación de hechos, por la omisión de noticias que no interesan directamente al reino y por el italiano usado con sintaxis bastante regular, que se aleja del lenguaje primitivo y lleno de dialectalismos napolitanos del texto original, aunque en tales «rifacimenti» abrevaron cronistas e historiadores de la época. Así el texto compilado y recogido por Muratori en Rerum Italicarum Scriptores se sirvió de una copia que le procuró el consejero regio Costantino Grimaldi, al que dedicó su obra?

El primero que habló de tal manuscrito fue Angelo di Costanzo en su prohemio a la Historia del Regno di Napoli ${ }^{8}$.

La crítica más rigurosa acepta que la transcripción cuidada por Faraglia para la Società di Storia Patria está hecha sobre el códice más antiguo, como ya se indicó, y que Bonincontro había sabido y usado tales Diurnali para sus Annales, cuando en 1456 había conocido a Alfonso V de cuya amistad, estima y afecto gozó?

Muratori transcribe en italiano «legibles y lo hace sobre arifacimenti» "ex codice manu quondam exaraton de Valletta ${ }^{16}$.

5. "Diurnali detti del Duca di Montelione", nella primitiva lezione, di un testo a penna posseduto dala Societa Napoletana di Storia Patria. Publicati a cura di Nunzio Federico Faraglia (Società Napoletana di Storia Patria). Napoli, Tip. Giannini e Figli, 1895.

6. CAPASSO, B. Le forti della Storia delle provincie napolitane dal 568 al 1500 con note e un copioso indice alfabetico del Dr. O. Mastroianni. Napoli, R. Margheri.

7. MuratoriUs, L.A. Rerum Italicarum Scriptores, Mediolani, 1732. Ex. Typ. Societatis Palatinae. Tomus XXI, Diaria Neapolitana ab anno 1366 usque ad annum 1478. Italica rudi lingua conscripta, Auctore Anonymo, nunc primum afferuntur in lucem e Ms Codice Nobilis Viri Francisci Vallettae, jurisconsulti, p. 1027-1109. Véase más adelante el „Prefatio* de Muratori...

8. Di Costanzo, A. Historia del Regno di Napoli. Napoli, Apud Cachio, 1582.

9. BoNinCONTRu, L., Annales ab nno 1360 usque ad annum 1452 nunc primum e M.s. codice Miniatense in publicam lucem prolatum, Rerum Italicarum Scriptores, Tomus XXII, p. 1-125.

10. Cfr. Praefatio de Muratori, traducido en páginas siguientes. 
El texto de Muratori es un "aggiornamento» gramatical, estilístico, sintáctico y hasta léxico del texto antiguo con el objeto de evitar confusionismo del mismo original sobre varios hechos descritos, a menudo, con sintaxis inconexa y con frecuentes dialectalismos, así como con pasajes oscuros, logrando con ello un compendio más claro y exacto de la narración, aunque también adultera la originalidad del manuscrito. Parece ser que se sirvió y ayudó de copias retocadas en el siglo XVı. No hay duda de que aclara nombres, fechas, ambigüedades, aunque contamina y entiende a su manera el texto antiguo.

El «M.s Montelione» trata en su primera parte (que no se traduce) de una monótona y sucesiva anotación de nacimientos, número de hijos, matrimonios y muertes de reyes, elecciones y muertes de papas, etc.; el manuscrito sólo comienza a tener visos de narración cuando aborda vicisitudes trágicas y tumultuosas del abigarrado reinado de Juana I.

Solamente con la descripción, casi novelesca, de la conquista del reino de Nápoles por Alfonso V de Aragón, los Diurnali se convierten en asunto interesantísimo por las noticias del día a día, por anécdotas de primaria humani$\mathrm{dad}$, por las refriegas entre las partes contendientes, por el desarrollo de las gestas y acciones de guerra y de ejemplares y caballerescas actitudes, no desprovistas de recelos, desconfianzas, insultos, "vendetten, ejecuciones sumarias y brutales...

Al final del manuscrito hay un Anexo, que recae en el vicio de anotaciones, muertes, matrimonios, fiestas, calamidades, aunque la sola descripción de la caída de Constantinopla eleva el interés de éste al grado máximo.

Un estudio someramente lógico de temas nos puede llevar a la conclusión de que el autor fuera un contemporáneo que, en la primera parte, usa datos recibidos y los reseña notarialmente y que posteriormente cuenta, como testigo presencial, los hechos que va narrando. Unos datos los sacaría de la tradición oral popular, que sería su primera fuente, y que él contrataría y aclararía en errores de fechas, hombres, lugares, etc., y los otros ya tienen la vivacidad, personalidad y participación psicológica del autor-testigo de lo narrado, como cuando anota una experiencia personal respecto a un hecho de armas y traición... ket ad me non volse ascultare Santo Parente, un homo d'armi, che mai non haveria perduto lo castello ${ }^{11}$ o cuando da sus juicios personales sobre vicisitudes y actitudes de los varios contendientes, condottieri, soberanos... y se contrista al ver el reino angevino ${ }^{12}$ de Nápoles en mano de dos hombres como Caldora $y$ Sforza de los que dice «non si trova havere servuto nè Dio nè

11. DDM, AD. 1423, 11 gennaro.

12. Los trece hijos de Carlos II: Carlos II de Hungría; Ludovico, obispo de Tolosa; Roberto, rey de Nápoles; Felipe, príncipe de Tarento; Raimundo Berlingheri, conde de Andria; Juan Tristano, muerto niño: Juan, príncipe de Acaia y duque de Durazzo: Pedro, conde de Gravisa; María, casada con Sancho de Aragán y luego con Jaime de Aragán, sen̂́or de Jericó; Margarita, mujer de Carlos, conde de Valois; Beatriz, mujer del marqués de Ferrara, Azzo IV d'Este y luego de Bertrando del Balzo, conde de Montecaglioso y de Andria; Eleonora, mujer de Federico II, rey de Sicilia. 
lo Diavolow, dando siempre una impronta muy personal a los diversos momentos y comportamientos objeto de su narración ${ }^{13}$.

\section{Confrontaciones, calcos y ampliaciones}

2.1. Los Diturnali del Duca di Montelione comienzan narrando la concesión del título e investidura del reino de Jerusalén y Sicilia, (que entonces tenía el rey Manfredi, hijo de Federico II Barbarroja), hecha por el papa francés, Urbano IV, al rey Carlos d'Anjou, hermano de Luis, rey de Francia.

A continuación se habla notarialmente de una serie de papas, de su ascenso al trono pontificio, muerte y sucesiones desde Clemente IV a Gregorio X, Inocencio V, así como de la sucesión de Carlos II, tras la muerte de su padre Carlos I.

En 1287 muere Inocencio V. Juan II sucede a Adriano V cun mese e poco piú provai come pesa il gran manto a chi dal fango il guarda Nicolás IV, Celestino V (único papa que renuncia al trono, el 13 de diciembre de 1254), permitiendo así la sucesión de Bonifacio VIII de la familia Gaetani.

De 1238 es la batalla entre Carlos II y los catalanes: el rehén Roberto, hijo de Carlos II, se casa en segundas nupcias con Sancha de Aragón, hija del rey de Aragón.

En 1322 muere Carlos II y le sucede el rey Roberto. Se canoniza a Tomás de Aquino y a Luis de Francia, hermano del rey Roberto (1317).

En 1316 la hija del rey de Austria, viuda del emperador Arrigo VII, se casa con el duque de Calabria, hijo de Roberto. Muere la primera mujer del duque de Calabria, que se casa de nuevo con María de Valois, primogénita del segundo matrimonio de Carlos de Valois y Margarita d'Anjou, hermana de Roberto. De tal matrimonio nace Ludovica (muerta poco después) y Juana, la futura reina, Juana I. El rey Roberto casa a su nieta Juana con el segundogénito del rey de Hungría, Andrés. En 1342 muere el rey Roberto. Es estrangulado en Aversa el rey Andrés y la reina Juana se casa con Micer Luis de Tarento.

Para vengar la muerte del rey Andrés, su padre, rey de Hungría, viene a Nápoles con un ejército de más de seis mil caballos y, entre otros objetivos, hace cortar la cabeza al duque de Durazzo, que le había robado la mujer.

En 1362 muere el rey Luis y su viuda, la reina Juana, se casa con Jaime de Aragón, infante de Mallorca.

En 1375 muere Urbano papa y en 1382 muere Juana I, que es sepultada en Santa Clara de Nápoles.

La reina expulsa a los franceses tras conquistar Castel Nuovo a su marido, jefe de la conspiración, al que hará encarcelar dos años. 
Muere el papa Gregorio y es nombrado el Colonna Martín. Ante el papa Martín va el antipapa "Gioanne", «era venuto in Fiorenza ad obedire a Papa Marino"; Juan XXIII es depuesto por el Concilio de Costanza, lo que da fin al Cisma de Occidente.

Las amenazas al reino desde 1388 por parte de la Casa d'Anjou encontraron respuesta contundente en el rey Lanzilao que en 1399 echó al duque d'Anjou y reinó incontrastado durante 15 años.

Se hace una detallada descripción, bastante confusa, de este período de contrastes y de alterno dominio de la Casa d'Anjou y Durazzo. «Et po venne in tempo di re Lansalao et cacciollo da lo Reame et giosende per fatti soi. Et questo Duca d'Angioia signorizo in questo Reame anni $\mathrm{XI}$ et intro gli 12, et dopo lo Re Lansalao signorizzo tutto lo Reame intero 15 anni: et dopo fo morto lui et la madre, et remase la regina Gioanna secunda que ha signorizato lo Reame fino a mo ch'e venuto lo Duca d'Angioia ad invadere suo Reame anni cinquex. Muratori, más claro, dice: angradito il Re Lanzilao lo cacciò et esso restaje Re di tutto a li 1399 et regnnò nel Regno integro Re Lanzilao, dopo la cacciata di Re Loise, anni 15. Il terzo fu figlio del secondo e nepote del primo Duca [d'Angiò]; venne a fare guerra a la Regina Giovanna II nell'anno $1420 \ldots$...

2.2 Reanudados los ataques con Luis II d'Anjou, la reina Juana II en 1420, tras la adopción de Alfonso de Aragón como hijo y sucesor del reino, éste acude en su ayuda con 12 galeras, 5 galeotes ututti con catalani et spagnuoli $\%$. Las naves del duque d'Anjou, apenas divisan la armada del aragonés, «sende partero et le galere gero a Castello a Mare»: era el mes de septiembre de 1420. Comenzaba la épica conquista del reino de Nápoles por parte del rey de Aragón. En tal ocasión la reina entregó el Castillo dell'Ovo kin mano ai catalani a loro petitione per parte de lo figlio ottivo Re de Raona (ali 6 de settembro anno Domini 1420. 14 Indizione)».

El 19 de septiembre, tras la adopción de Alfonso por parte de la reina Juana (Alfonso tenía 24 años), le puso en posesión del Ducado de Calabria; tal día Alfonso cabalgó triunfalmente por todo Nápoles, vestido de paños de oro y con las cuatro banderas, del papa, de la reina, de la Iglesia y de Aragón. Hubo luminarias en la ciudad... DDM así lo describe: "Li 19 de settembro... la regina Gionna mese in possessione lo Vice Rè de rahona suo figlio de lo Duccado de Calabria et questo di cavalcó questo Vice Rè per tutto Napoli con gran honore e triunpho et lui vestito de drappe d'oro et sopra la testa quattro bandere l'una all'arma de papa Colonna, l'altra della Chiesa, l'altro per tutta all'arme de la Regina con quella de Rè de Rahona quartata et l'altra de lo Reame... Ali 24 de settembro la regina Gioanna seconda fece jurare humagio a tutto Napole tanto ali gentil homini quanto a lo Populo in presentia de lo Vice Rè de Rahona, et lo jurare fo in questa forma, che mentre la 
regina vivea possedesse suo Reame tutto reservato lo Ducato di Calabria et dopo sua morte fosse tutto lo Reame de suo figlio Rè de Rahona et questo Rè de rahona lo devea agiutare et defender contra ogni personas).

Zurita lo narra con una pequeña diferencia: las banderas eran llevadas por Don Ramón de Perellós, a quien el rey hizo su «Lugarteniente General en el Ducado de Calabria y en los Castillos... El mismo día anduvo Don Ramón de Perellos con gran triunfo, con quatro banderas, con las armas del Papa y con las insignias Reales de la Reyna y del Rey a quarteles: y se le entregó el castillo del Ovo y dentro de cinco días hizo dar la Reyna los homenages de las Congregaciones del regimiento de aquella ciudad que está en poder de los gentileshombres, que ellos llaman Sejos y del Pueblo de Nápoles, en presencia del Visorey, don Ramón de Perellos, y juraron que en la vida de la Reina, la tendrían por su verdadera Reyna y Señora, que poseyesse todo el Reyno, excepto el Ducado de Calabria, que habría ya renunciado, y después de su muerte obedecerían al Rey de Aragón, su hijo, por verdadero Rey y legítimo sucessors.

El inspirador de la política proaragonesa en la Corte de Juana II fue Antonio Carraffa «Para esto fue buen Ministro aquel Antonio Caraffa, que los catalanes llamaban Malis Carraffa y con mucha disimulación... con el Embaxador García Aznar passó a Cerdeña y halló al Rey en Alguer... Quando llegó Carraffa al Rey y hizo el oficio que se requería para representar el peligro en que la reina estava y todo su reino y persuadir que con ánimo valeroso se tomasse la empresa de amparar a la Reina de sus enemigos, que era obra de Príncipe tan excelente, como é era el Rey con su ánimo muy generoso, no se movía tanto por la esperanza que se ponía delante, que sucedería en aquel reino, como hijo y único defensor de la Reina ni por el derecho que se le ofrecía de ponerle luego en la possessión del Ducado de Calabria como a legítimo sucessor....".

Solicita la reina Juana a Alfonso ayuda urgente, dado que Luis ampliaba su dominio en Nápoles; pero los consejeros sicilianos de Alfonso le desaconsejan tal empresa. Según DDM «Ali 5 di Marzo... in Napoli si ragionava come in Sicilia Messer Bernardo in Calvera e certi altri gran signori de Sicilia consigliaro a Ré de Rahona... che lo detto Rè non devesse venire a questo... havea fatto piú tradimenti ali antecessori et alo padre de Rè Loise et cosi anchora li signori de lo Reame...n, que Zurita con más detalles y mayor profundidad relata de la siguiente manera: a...deliberación de los de su Consejo. Avía bien que considerar para que el Rey no se pusiesse ligeramente en una empresa como ésta... que no se engañasse el Rey por las vanas promessas de los Barones, como fue burlado el Rey Luis su Agüelo y que él y su padre por la misma livianidad avían sido echados del Reynom, donde revierte las acusaciones, que DDM hace directamente a la reina Juana sobre los barones del 
reino, dejando a la institución monárquica libre de culpa e intacta en los manejos y sospechas que iban surgiendo al respecto. No será la primera vez que el cronista del reino inclina ligeramente el plato de la balanza de la parte regia, sea donde sea que éste se halle, amigo-aliado o enemigo y adversario.

La vacilación de Alfonso hace que la reina, ache tenea le mani in doe pasten, comience a acordarse con el de Anjou y sólo la venida de las naves del aragonés en su socorro la hace abandonar el veleitario cambio para volver a su primera alianza. El Maestre de Montesa, embajador del rey, ya había asegurado a la reina que el rey de Aragón usenza nullo fallo ipso será prestissimo a Vostra maestá a fare tutte quelle cose seranno grate a vostra Maestá... et la Regina per la venuta di questo Mastro di Montese nde mando a Messer Bernardo Archamone exclusom, y asi el embajador angevino Arcamone se ve excluido de posibles pactos a favor del rey Luis por el subitáneo cambio de la reina Juana. Zurita recalca: «Tratando la Reyna de concertar con su enemigo, llegaron a Iscla quatro galeras que el rey embiava en socorro de las cosas de Nápoles, entretanto que su armada real se ponía en orden... y mandó certificar a la Reyna que brevemente ponía en orden su partidan.

2.3. El 7 de junio Braccio de Montone «venne a lo soldo de la Regina et de Ré de Rahona con cavall tre milia et come intrò in terra de lavore piglio Marigliano et po venne in Napole et stette ad Ygliulo de che la Regina et Napole ne fecero gran festa.... .

Braccio de Montone entra a formar parte del bando aragonés y es celebrado con gran fiesta. Zurita es más explícito en el razonamiento de la asunción de Braccio: «...el Rey deliberó de traer por Capitán de su exército, con parecer de la Reyna, a Baccio de Montone de Perosa, que era muy excelente capitán y fue muy estimado de la nación Italiana... para que saliese en campo contra el Duque y se començasse la guerra con autoridad... Era a los siete de junio deste año quando Braccio fue a tomar cargo del exército al sueldo de la Reyna y del Rey y tenía tres mil cavallos y entró con tanta celeridad y tan repentinamente, que no se le pudo defender la entrada ni resistir por Sforça, aunque se puso en ello y le salió al encuentro y, entrando en tierra de Labor, tomó Marigliano y de allí se entró en Nápoles. Aviéndose detenido Braccio diez días en aquella ciudad fue sobre Castellamar de Stabia por orden de la reyna y entró de noche por combate y puso el lugar a sacon, en lo que sigue a DDM, donde dice "Ed essendo stato lla due di, piacque ala Regina andasse a Castello a mare de stabia. Et pigliolla per forza e mesela a sacchon.

La noticia de Braccio al servicio de la reina Juana mobiliza a Alfonso: Ali 25 de Jugno, sapendo Rè de Rahona che Brazo era gionto ali servitij de la Regina, subito se parte et venne con otto nave et sidici galere et tra bergantini et altre fusti che foro 27 , donde la Regina et Napole ne 
fecero gran festa et luminarie... et come gionse stette allo Castello dell'Ovo una notte... et poi trasio in Napole con lo palio come Rè et po scavalcò alo castello nuovo et fecero reverentia ala Regina et pigliolo molto charo come figlion; Zurita, entre otras divagaciones gloriosas, recoge en lo sustancial el pensamiento de DDM: «Teniendo el Rey en orden su armada, que era de diez y seis galeras y ocho naves y otros navios menores, salió del puerto de Mecina a veynte y cinco de junio, aviéndole llegado la nueva que Braccio estava en campo, haziendo la guerra en su nombre, y passó el Rey con su armada a Iscla... y atravesó el castillo Nuevo a hazer reverencia a la reyna y recogió al rey con grandes muestras de amorn.

2.4. Primeros síntomas de desavenencias inducen al papa Colonna a enviar legados para hacer paces entre la reina, el rey de Aragón y el duque d'Anjou. De una parte el angevino y Sforza, de la otra el aragonés y Braccio. Zurita especifica que los «dos Legados Apostólicos (mando) al de Sant'Angel, que era espańol, y fue creado por Benedicto, al Rey; y al de Flisco, al Duque de Anjous, con plática de medios de paz... y bolvieron sin ninguna buena resolución... y dividiéronse los potentados de Italia en parcialidades de Anjoínos y Aragoneses» que DDM más sucintamente: «... Lo Papa mandó in Napole lo Cardinale di santo Angelo legato et lo Cardinale de lo Fisco... a volere contrattare et ponere pace entra la Maestà sua et quella de Re Loise et Re de Rahona in Napole e stettero certi giorni a mettere questa concordia et per nullo modo non pottero et andaro sende exclusi senza nullo accordio...».

Consecuencia de primeras desavenencias y choques entre las facciones antes aliadas es la toma de Acerra: "En este tiempo creciendo más las sospechas que el Duque y Sforça tenían de Tartaglia le mandaron cortar la cabeça en la plaça de Aversai, dice Zurita, recordando el episodio de la culpabilidad de Tartaglia en el asedio de Acerra por Alfonso siguiendo a DDM... une fo pigliato sospetto per Sforza e per altri Tartaglia de Lavello, sichè per questo le ne fo tagliata la testa alla Città di Aversa».

Estos roces manifiestos entre la reina y Alfonso de Aragón se confirman a raíz de la toma de Sorrento y Massa, que el rey de Aragón edicea volerla ipso et la Regina la volea essa. Ultimo restaro a Rè de Ragona», lo que comporta "que Intra la Ragina... et Rè de Rahona fo una gran divisione et principio de inimicitia y el acercamiento de la reina a Sforza que el rey de Aragón ve con malos ojos, «per la supradetta occulta nemiciria de la Regina a Rè d'Aragona", que Zurita recuerda "Ay Autor que afirma que, queriendo el Rey que estos lugares se le rindiessen a él y no a la Reyna, començó a descubrirse entre ellos la mayor sospecha, de que nacio la discordia, que se declaró ser de gran odio y enemistad...., que recalca más adelante "tuvo a lo que se juzgava, esta gran dissension su principio de averse puesto en la obediencia del Rey los Lugares que se le rindieron en la montaña de Sorrento...». 
El rey aprovecha para ir de caza a etirare quaglien; aquí se produce la escena del rey que cae del caballo y Sforza que acude rápidamente a ayudarle "con molta destrezza", lo que comporta de parte del aragonés ánimo benévolo hacia Sforza, quien, a su vez, le promete sojuzgar a todos los partidarios del angevino.

La reina, no del todo convencida de las buenas intenciones de Alfonso, en secreto contacta con Sforza: a...la Regina confortava lo ditto Sforça et ... imponevale occultamente che confortassi tutti signori che teneano de la banda de re Luisen; la razón de tal cambio nos la ofrece también Zurita: whaberse puesto en la obediencia del Rey los lugares que se le rindieron en la montańa de Sorrento, de que tuvo mucho descontentamiento el Gran Senescals.

Escaramuzas varias entre la Corte de la reina Juana, su senescal y el rey de Aragón crean zozobra de parte de la reina: "Passó la Reyna de Gaeta a Prochyta, como que iba a recrearse, dissimulando su miedo y deteniéndose allí algunos días; fuesse a Puçol para entrar en Nápoles. De Aversa se fue el Rey a Nápoles a su Palacio Real, que era el Castillo Nuevo: y dexó ordenado que la Reyna fuesse por mar y supo ella que a los Capitanes de las galeras se dio orden que la llevassen al mismo castillo donde el Rey estava, $y$ con aquel temor se fue por tierra, sin que se entendiesse, al castillo de Capuana que ella tenía su guarda... De allí adelante se fueron descubriendo el odio y rancor que tenían el Rey y la Reyna y sus Privados, que el Gran Senescal no quiso ir al Castillo sin su seguro... y el Rey para que pudiesse ir seguramente le dio su salvaguarda, escrita de su mano, con un sello de oron, tal cautela de parte del senescal que así describe el original DDM: «... et cosi lo Gran Nuovo volse securità et autentico salvo condutto da lo detto Rè de Rahona, lo qualo li lo fece come volse a bulle d'oron.

Hasta la mera preparación de unas justas con carros alegóricos en esta ocasión de grandes perspicacias era motivo de reflejar la rivalidad entre ambos bandos, pues se pensaba que de haberse celebrado, hubieran evidenciado la enemistad de unos contra otros... De hecho dice el autor de los DDM que el rey de Aragón tenía pensado coger prisionera a la reina, que, avisada por un espía, llama a Sforza en su ayuda: «... questi giorni vedendosi la Regina ad tal partito scrisse et mando uno speciale misso a Sforça suo compare, lo quale stava a Benevento, ...che devesse venire ad aiutarla dal ditto Rè de Rahona... et dapo l'altro dì sequente venne Sforza et cacciò la Regina da lo castello de Capuana et portolla a Nola et lla la lassò et ipso tornò alo Campo vecchio, puro per dare ordine et socorrere lo Castello de Capuano, alo quale era rimasto uno condestabile de infante con cento paghe et santo parente... lo che detto condestabile non fece lo devere... Sforza havendo avuto informatione di santo parente... do questo condestabile che non havea voluto far lo devere subbito esso con li soi mani proprii lo impiccò per la gola..... Collenuccio confirma: «... fece caricare tutta la robba de 
la Regina e lei condusse fuora di Capuana e menolla a Nola, drietto ala quale piu di cinque mila napolitani, maschi e femine che la amavano....n.

Zurita sigue línea a línea a DDM: «Fue otro día por la Reyna y sacóla del castillo de Capuana y llevóla a Aversa y después a Nola y volvió con una celeridad increyble a Campo Viejo por socorrer el castillo de Capuana, adonde avía dexado al Capitán Gracián con cien soldados y a Santo Parente... pero el de Capuana se rindió al Rey a partido con tanto pesar y sentimiento de Sforza que por su propia mano ahorcó a Gracián, porque no hizo su dever como Santo Parente quisiera.

2.5. De la batalla que hubo entre angevinos y aragoneses dice Zurita: «Llegó (Sforza) con su Campo a un lugar que llaman Santa Maria de Ogliulo, a treinta de mayo, y allí supo que el rey avía mandado salir fuera de la ciudad toda su gente, que eran hasta tres mil de cavallo y de pie. Y Sforza, según escriven, no tenía más de seyscientos de cavallo y mal en orden... ordenó sus esquadrones, como mejor pudo. Pidiendo los suyos... qué apellido tomarían, dixo: "Herid a los bien vestidos y bien a cavallo", porque los suyos estavan en cavallos muy flacos y ellos mal vestidos... A los primeros encuentros perdió Sforza muchos de los suyos y los demás ya rebolvían a ponerse en salvo... Ay Autor catalán antiguo, que afirma que salieron de Castillo Capuana trescientos hombres de armas con quatro mil de a pie del pueblo e hirieron en los nuestros por las espaldas y entonces se desbarataron y con este socorro Sforza arremetió contra Cico Antonio, que llevaba el estandarte Real y se lo quitó de las manos y alli fue preso y, viendo esto los suyos, cobrando nuevo esfuerzo, pelearon animosamente y los nuestros no podían pelear ni tampoco recogerse y muy pocos resistían la empresa de los enemigos". (Este autor catalán antiguo siempre puede ser un espańol o italiano de la facción aragonesa, y que se puede identificar con el autor anónimo de los Diurnali, que reseńa el hecho del siguiente modo: spenultimo maggio... Sforza sentio come Re de Rahona havea cacciato fora tutta sua gente d'arme, quale era in Somma, de circa tre o quattro milia persone, intra cavalli et ad piedi, et Sforza non havea se non circa 200 o 300 infanti male in punto. Sforza per ajucare la Regina soa commare si posse volerse mettere in tanto pericolo... et ordino soe squadre come se pottè de che la gente d'arme de Sforza adomandaro che insignale havemo et esso rispose che feressero ali ben vestite et ben a cavallo, perche loro stavano male a cavallo rugilenti et male vestite et questo fo l'insignale et Sforza scese a casa nova dove trovó la gente de Ré Rahona che aspettavano et subito Sforza fece dare dentro per la prima afficcata; la gente di Sforza hebbero una mala rotta, de che Sforza vedendosi male arrivato, si posse in disperazione et corse et piglio detto Cicco Antonio per petto, ...teneva lo stendardo de Ré de Rahona et in effetto lo prese presone et levolli lo stendardo... per virilitá del detto Sforza, la gente 
de Rahona si mese a rotta et íncominció a fugire de che ne foro presoni assai di quelli signori catalani et jtaliani et homini di Napole et forone per quella giornata morti piú de ducento corseri grossi...m; como consecuencia de esta victoria sobre el aragonés, la reina manda regresar a los desterrados *quelli che erano con Re Luise*.

2.6. El 11 de enero de 1423 llegan naves a Nápoles, según Muratori. Zurita: usalió esta armada de la playa de Barcelona a onze del mes de Mayo y, según parece en Autor antiguo de las cosas del reyno y natural dell, fue de veinte y dos galeras y ocho naves gruesas: $y$ arribó al puerto de Nápoles a diez del mes de junio... Dezíase publicamente en Nápolesı, en lo que es fiel una vez más a DDM: «Et ali XI Gennaro presentis anni vennero in Napoli otto navi grosse et galere 20 , o vero 22 , et dicese che vennero per volerne portare la Regina, perché Re de Rahona non se credea essere Ré signore integro, se non havea la Regina entro sue mano". Atacan la ciudad los aragoneses y oordenó con los Capitanes de las naves y galeras que se combatiesse la Ciudad... fueron del todo lançados para dentro y cogidos... Embiaron los Napolitanos a llamar a Sforza para que los socorriesse y sacasse a la Reyna y no vino aquella tarde y llegó otro día por la mañana y halló perdida la plaça del puerto hasta Santa Clara y puesto todo aquello a saco... y todos los grandes, que habian ido a visitar a la Reina, llenos de miedo. Salió el Rey otro día al amanecer con sus hazes en ordenança y combatióse la ciudad por mar y por tierra... mezclóse una muy rezia batalla con la gente que había puesto la noche passada en la defensa de aquellas calles, resistiendo Sforza con gran valor y con un vigor de ánimo terrible, segin se afirma.. Púsose fuego por diversas partes de la ciudad...y, no pudiendo resistir el impetu y furor de la gente del Rey, se fueron los enemigos recogiendo... el (castillo) de Capuana se rindió al Rey a partido... y con tanto pesar y sentimiento de Sforza, que por su propia mano ahorcó al Gracián", que traduce literalmente "con le sue mani proprio lo impiccó per la gola».

Concluye Zurita afirmando que ucon esta victoria quedó el Rey señor de aquella ciudad y de los Castillos».

A Sforza, el rescate de los rehenes en su poder le proporciona 2.000 ducados, según DDM: «Et Sforza li diede tanti signori allora, li quali se haveriano riscossi più di doi milla ducati, li quali presoni foro messer Bernardo Perigliuso, messer Joanna de Moncata, messer Bernardo Santigila, messer Bardascin, messer Incoglio, messer Gurillo, Raimundo de Moncata, messer Federico de vinti miglia et Conte Herrico, lo conte Joanne de Ventimiglia et piú alti adherenti de Rè de Rahonas.

Zurita dice que la reina Juana «... dio entonces a Sforça todos los principales prisioneros de que pudiera aver un gran rescate... diéralos a todos de mejor gana por solo el Gran Senescal, y el Rey le mandó poner en libertad por Don Bernardo Centellas y Don Ramón de Perellos». 
2.7. El 11 de junio la reina envía embajadores a Roma y propone al rey Luis llegar a un acuerdo. El rey Luis acepta y es recibido en Aversa con toda solemnidad. El rey de Aragón en principio trata de disturbar tal concierto "tutto facea per volerlo gabare et Rè Loise lo conoscette et piglio lo partito della Regina et partiosi da Roma et venne ad Aversa... et messer de Tropea, lo quale all'hora stava con la Regina... fece lo sermone, lo quale nel principio respose: vere filius Dei est iste..., si DDM dice de las aviesas intenciones del rey de Aragón respecto al rey Luis, Zurita recalca que «embió al Duque un su privado Duque con esperanza que se concertaría con él... y, sospechando el Duque que se hazía por entretenerse y engañarle, porque la Reyna no se fiasse en él, se concertó con la Reyna y fue luego a Aversa y... el Obispo de Tropea, que estava con la Reyna, tuvo una larga plática de alabança del Duque, tomando por fundamento de su sermón aquellas palabras de la Sagrada Escritura: Verdaderamente éste era hijo de Dios.....

Ante tal acuerdo-alianza ya estaba el rey Alfonso preparado para hacerse a la vela rumbo a Marsella y Catalunia; llama a Braccio de Montone, que manda a Antonio Caldora y a otros capitanes a defender las posiciones conquistadas «...et venuta questa gente Re de Rahona propose de voler sende andare in Catalognia con tutte le navi et galere, ... et volendosi partire lo detto Rè de Rahona, Sforza et Rè Loise con tutta la gente d'armi vennero infino ala Magdalena a mostrare a Rè de Rahona come Rè Loise era bene in accordio con la Regina; et Rè de Rahona vedendo questo fece uscire tutta la gente d'armi soa, cioè messer Jacovo Errico et Bernardino et altre genti d'arme catalani et Italiani et galioti de l'armata et uscero alo ponte per havere ad fare con Sforza, de che Sforza diede dentro ad tutta questa gente di Rè de Rahona, la quale era a duplo più quella de Rè Loise et per forza d'arme le caccio fino dentro Napole et ipso Sforza pose lo stendardo suo de lo Diamante per fino alo Burgo nuovo, di che Rè de Rahona, vedendo questo ne fo in grande pensiero vedendo tanta gente cacciare tutto la gente sua per forza d'armen; Zurita transcribe casi literalmente a DDM: «... y travóse con la gente que salió de la Ciudad una muy rezia escaramuza. En la qual, ay Autor natural del Reyno, de aquel tiempo, que no se nombra, que afirma que los nuestros fueron rompidos y lançados dentro de la Ciudad por fuerça de armas y que Sforça passó a poner su Pendón del Diamante, que era su divisa, hasta el Burgo nuevo y que este caso que dio mucho descontentamiento y cuidado al Rey, viendo los suyos lançados de mucho menor número de gente».

Al pasar por Marsella la pone a saco, se lleva consigo el cuerpo de San Luis de Francia hasta Cataluña "possila a sacco magno... et portaronde lo corpo de san Loise de Franza in Catalogna». Dice Zurita: «Avía mandado el Rey en la furia de llevar a saco aquella Ciudad,... que se procurasse de aver el cuerpo de San Luis, obispo de Tolosa... como la más preciada joya de aquella ciudad... por suceder de la Reina Doña Blanca, su Hermanas. 
2.8. Un mes antes de la muerte de Sforza, que se ahoga al querer salvar a un "galuppon de su gente de armas, toma Gaeta, Prócida y Castellammare "De la guerra que huvo en el Reyno entre el Duque de Anjous y el Infante don Pedro y cómo los Anyoínos se apoderaron de las ciudades de Gaeta y Nápoles y del Castillo de Capuana" lo que comporta «fo tagliato a pezzi Joanne de Valenza, un catalano molto captivon. Zurita afirma que ifue degollado un cavallero valenciano, llamado Juan Catalán, que la tenía en defensan.

2.9. En un sucesivo cerco de Nápoles, el infante Pedro, hermano de Alfonso V, «y Guido Torello con su armada asseguró lo de la mar y echó la gente a tierra y huvo diversas escaramuças entre los Anyoínos y Aragoneses, pero los de la ciudad peleavan de tal manera que las más de las veces venían a habla con los enemigos. Desto se alteró e indignó tanto el Infante, que estava en el Castillo Real, que según afirma un Autor antiguo de aquel tiempo y de aquella nación, quiso mandar poner fuego a la Ciudad, y ya venían en ello todos los de su Consejo, con una cruel desesperación diziendo que era mejor el lugar abrasado que perdido, y sólo Cola Suttil y Iacobo Caldora fueron de contrario parecer. Dezía Caldora que ni él ni ninguno de su linaje avían edificado jamás una tan hermosa Ciudad y assi no quería hallarse al deshazerla, antes suplicava al Infante que, si tenía ánimos para dar lugar que se hiziese una tan gran crueldad, se lo avisasse algunos días antes, porque no quería ver de sus ojos un tan abominable acometimiento; y el Cola añadía que por ventura de tal hecho como aquél desplazería en el corazón al Rey, que mostrava por su Real ánimo y clemencia mucho arrepentimiento por lo que se havía executado en Marsella... y les avía encomendado aquella ciudad con orden que la defendiessen y guardassen y no para que la abrasassen. Con estas y otras razones afirma aquel Autor antiguo que desviaron al Infante de un tan bárbaro y desesperado propósito, mayormente que se tenía esperança que el Rey tornaría muy presto o embiaría tal armada que pudiesse ser muy superior a la de sus enemigosn; el texto del «Autor antiguo» de los Diurnali: «... et vennece Capitanio Guida Torello... donde vennero una gran quantitá de lombardi... et fanti assai et le navi et le galere per mare et ogni giorno faceano scuntri de lanza con Napoletani. Et per questo haveano parlamento insieme et facevno trattati de fare entrare Ré Loise, lo fante, sentendo et avvisandose de questo... Et appresso ordinario combattere lo Castello Novo dove era dentro lo fante. Et innante se combattesse lo detto castello de Capuana, lo detto infante se dispose totaliter volere ardere Napole. Et per non fare de sua testa se lo misero in consiglio et tutti signori italiani et siciliani si trovaro conforme in uno dire et tutti dissero che lo Infante dicea bene che Napole se ardesse, perchè migliore valea terra disfatta che terra perduta, salvi due, li quali restaro dietro a dire; l'uno fo messer Nicola Sottile et fo palermitano lo quale disse che per nullo modo ad esso parea che una 
terra cosí fatta come è Napole et de tanta nobiltà si devesse ardere et anchora Rè de rahona I'havea lassata in guardia de lo Fante et a tutti voi signori che vedendo soa Maestà et trovandola arsa, donde voi credissivo havere meritato credendovi havere ben fatto; forsi averissivo mal merito et disgratie. Anchora noi aspettamo l'armata del signore Rè de Rahona... Et Messer Iacobo Caldola affirmó tutto lo dire de messer Nicola, aggiungendo esso anchora et dicendo che mai nullo de soi antecessori havea fatta sí bella città et per questo esso per nullo modo sence volea trovare per doi ragioni, l'una per non fare tanto streminio et damnagio, Paltra perchè Rè de Ragona, meritamente havendo fatto questo, sende potria render mal merito donde esso l'aspettava buono, ma, si puro lo signore infante si dispone de lo fare, prego dicitemelo dui dì innante, perchè me ne volglio andare, che innante vorria norire che me trovare ad tal desfalta, de che lo signore infante et altri, audendo lo dire di questi doi et parendo che questi dicessero bene, determinaro in tutto lassare loro male proponimenton.

2.10. En la batalla de Nápoles que ganaron los Anyoínos, Caldora decide traicionar y hacer entrar a los invasores bajo pactos y se rinde wa patti, salve le persone e la robban; dice Zurita que "Caldora no andaba firme en el servicio del Reys, en lo que concuerda con DDM: a... et in questo accordio nce foro messer Jacobo con Guido Torello de darli Napoli. Si volse de patto che tutta la gente et la robba de napoletani fosse salva, reservato robba de catalani... Messer Jacobo li aperse la porta de lo mercato et fece intrare, da che vedendo la gente d'arme de Rè de Rahona si mesero in fuga et... ne foro presi assai catalani et intra l'altri nce fo preso Joan de Calata, che era all'hora capitanio a guerra in Napole, lo quale si riscosse sei milia docati»; Muratori aclara y amplía a DDM: «Al fine Messer Iacobo patteggió che riceveria tutte le paghe della Regina, che li dovea dare Re Alfonso e che Napoletani e loro robbe sariano salve, lasciando ad arbitrio de' soldati quelle de' catalani e aperse le porte alli 12 de aprile del 1424", versión que confirma Zurita al reconocer "pensó [Caldora] en lo que le convenía y lo cierto es que, según escribe Bernardino Corio, que siempre puso por delante el dinero a lo que era justo y honesto... y fue acordado que la Reyna le mandasse pagar todo el sueldo que devía a su gente y que los Napolitanos y sus haziendas fuessen libres, dexando a discreción de los soldados las de los Aragoneses; y siendo abiertas las puertas, entró en ella el exército de los enemigos. Esto fue a doze del mes de abril deste año y fueron presos la mayor parte de los Aragoneses y Catalanes, que se desmandaron de la gente de la armada y de los napolitanos mismos; y entre ellos Don Juan de Moncada, que pagó su rescate seis mil florines».

Los Anyoinos no logran tomar Castillo Nuevo y dell'Ovo, pues la armada genovesa de Torello, que eficazmente habia participado en tal batalla, acababa su contrato: «Guido Torello, havendo preso Napoli et 
fatto questo effetto, se partio perché le navi e galere erano assoldate solo per quello tempon, dato que recoge Zurita siempre de la fuente del "Autor Antiguon: "no llevaban más sueldo del que fue necessario para este tiempon.

2.11. En la defensa a ultranza de Aquila Braccio se debe enfrentar a los ejércitos de la Iglesia y de la reina Juana y del duque d'Anjou, aliados contra él. Braccio de Montone es cogido y herido mortalmente. Micer Jacobo Caldora se acerca al moribundo, que se confía a su piedad: "... Brazo semivivo aperse l'occhio et disse: o signor messer Iacovo, io te prego che me habbiate per raccomandato; messer lacobo disse: Io farró quello a te che haverisse tu fatto ad me, si me havisse havuto presone voltando le spalle et andosende via... lo detto Brazo, intendendo tale risposta e chiuse l'occhi e mai non parló piús. Era el 25 de mayo de 1424. El papa Colonna, Martín V, lo mandó sepultar fuera de Roma «l'impio et heretico... volea cacciare lo Papa et Regina Joanna et tutti li seguaci loro dal mondo... nel suo mestiere fo liale et ottenne sempre veritate", que Muratori remeda: "nel suo esercito era leale e valente".

2.12. La reina oye de una próxima llegada de la armada del rey de Aragón para uinvadere Napolin y reúne a los señores. El 20 de junio se avista la armada. La defensa de los napolitanos impide los objetivos del aragonés, que después de 27 días de estancia logra sacar del sitiado Castillo Nuevo al infante, dejando en su lugar a Dolman Carcirera.

El papa Martín Colonna pide para su sobrino nuevas tierras y obtiene de la reina Asturi y Nettuno enon finava mai cercare ogni giorno terre et castellaw. Muratori tilda al Colonna Papa Martin, ávido de tierras para sus sobrinos, de "non sazio".

2.13. El gran senescal, temeroso del posible afecto y simpatía que podía despertar en el pueblo el duque d'Anjou, le impide visitar Nápoles y hace que el angevino, al llegar a Nápoles, se tenga que ir a Calabria, que conquista a su obediencia: el gran senescal le aleja así de la administración del reino, gobernando como señor absoluto.

Gravisima falta de consideración del gran senescal a la reina, en cuanto cada día su poder acrecía; dice DDM: «salio in superbia et ira et usó alcune disconvenevoli et dishoneste parole ala donna soa Regina... piglióne molta turbatione, vedendosi venire da Donna a servas; el gran senescal, al verse negada su petición de tener el Principado de Salerno para regalárselo a su hijo en su próxima boda con la hija de Caldora, Madama María, lo que fortalecía su posición preeminente en el reino, de dominio total, "dominus dominantium" le llama DDM, había planeado una avisada política matrimonial que le lleva a dar una hija a Antonio, hijo de Micer Jacobo Caldora, y otra a Gabriel Ursino, hermano del prín- 
cipe de Tarento, los dos grandes señores del reino que podian contrastar su dominio; no contaba con la envidia de los cortesanos que aprovechan esta coyuntura de enfrentamiento con la reina y del disgustoso episodio de falta total de miramiento hacia la reina, su protectora y amante, para conjurar contra él, decidiendo su muerte en consejo secreto; a continuación van a la cámara del castillo Capuano, donde el senescal tiene aposento, y con el pretexto de la extrema gravedad de la reina le hacen abrir su habitación y lo rematan a hachazos y estocadas... «...lo Gran Senescallo attestò demandare più volte ala Regina Joanna lo Principato de Salerno, ne volea fare Principe Troiano suo figlio ... benchè lui signorizava et era dominus dominantium... la Regina non volse fare, lo gran senescallo salio in superbia et ira, uso dire alcune disconvenevole et dishoneste parole ala donna soa Regina, che l'havea fatto tanto magno, la Regina disdegnò grandemente...e occultamente con alcuni servitori soi et nemici delo gran senescallo ordino de lo fare prendere per ammonirlo de soi regugli in quello di 19 de Augusto...; lo gran senescallo si ando a dormire alo castello de Capuana, in una camera dove esso stava per stantia. Messer Ottinio Carazolo, la Duchesa de Sexa, Messer Francesco carazolo, Messer Pietro Paglione de Trane et tutti altri hebbero maturo consiglio, si non pigliano lo gran senescallo la Regina è donna de poca costanza, liberaralo ....per nostra sicurezza indicamo sia morto et fermandose in questo era l'hora iiij de notte venute, la detta Regina redusse ad executione lo fatto, tale modo comandó a squadre suo famiglio et servitore todisco che se l'havea menato da la Magna dovesse andare ad chiamare lo grande senescalco con dire che venisse presto che la Regina stava male et erali scesa quella gota da sua testa... lo gran Senescalco comanda a soi camareri... aprire la camera,... lui si posse ... in pressa a vestirsi... aperta la porta dela camera subbito entrò dentro messer Pietro Palagano de Trani et messer Francesco Caracciolo de Napoli, Urbano Cimino... et uno famiglio dela Duchessa de Sexa senza altro dire a colpi de stocchi et accette lo fornero et ammazzaro... La duchessa de Sexa era stato capo pede et principio de questa facenda, per quella notte insio fore de lo Castello, solea pernottare dentro lo castello come è parente de la Regina e andosende a Napoli... Messer Ottinio Caracciolo et Messer Marino Boffo, che era signore de Arienso, restaro dentro la corte de lo castello, si lo fatto non reuscia, proxime fugire; et referiti ala Regina, che era morto, linde sebbe male, et turbose molto et, indignandosi verso tutti, questi resposero non sende ha possuto altro fare, che lui se posse a gridare et a defesa; po la cosa fatta a li remedij; ....li soi parenti... subbito ordinaro mandare ad chiamare ad uno ad uno, de notte stavano per lloro stantie dentro Napole; veniti subbito, dice lo gran Senescalco, che la regina sta male et more. Et in questo modo andaro tutti et foro posti presoni... et la robba delo gran senescallo, del figlio, del Conte de Sant'Angelo, de alcuni altri soi parenti foro posti tutti a saccon. 
Zurita lo relata siguiendo al Autor antiguo, que cita para contrastarlo: «...y como quiera que él era señor absoluto de todo y lo governava y mandava a su alvedrío, con todo esto la Reynąno quiso dárselo ...de esto se indignó el Gran Senescal, que dixo algunas palabras muy feas y deshonestas ante la Reyna... y la Reyna, llena de sospecha y temor, recelando que, si no se refrenava la soberbia al Gran Senescal, no passasse a otras cosas peores, se estrechó con algunos de quien se fiava y determinó de mandarlo prender... y fue el día 18 de agosto, cuando se avía de celebrar la boda del hijo del Gran Senescal... el Gran Senescal se fue a dormir a una cámara del castillo de Capuana, donde tenía su aposento. Eran los principales de este acuerdo la Duquesa de Sessa, Otino Caraciolo de la Rosa y Pedro Palagano de Trani... si ellos prendían al Gran Senescal, la Reyna, que era naturalmente muy mudable, le mandaría luego poner en libertad y determinaron de matarlo. Ordenaron que a cuatro horas de la noche un Tudesco, criado de la Reyna de que hazía gran confiança y le avía llevado de Austria, llamasse al Gran Senescal y le dixesse que la Reyna estava muy fatigada de la gota, que le subió ya a la cabeça... Pidiendo el Gran Senecal de vestirse, entraron dentro los conjurados, que eran Francisco Caraciolo, Pedro Palagano y el Tudesco y un criado de la Duquesa de Sessa y le mataron a golpes de hachas y estocadas. No quiso la Duquesa, siendo la principal en un hecho tan grande como éste, hallarse aquella noche en el Castillo, aunque solía dormir en él como parienta de la Reyna, y Otino Caraciolo y Marino Boffa, que era señor de Ariano, quedaron dentro con deliberación de huirse, si el hecho no se executasse. De este caso se dolió mucho la Reyna, porque su intención no fue jamás de mandarlo matar, y los matadores se excusaron afirmando que no se pudo hazer de otra suerte, porque se puso en defensa y no era posible tomarlo vivo... y por poner remedio a la alteración que podía suceder de este caso, llamaron todos los parientes del Gran Senescal de su parte, deziendo que la Reyna se moría y assí fueron todos, que eran presos... y sus casas se pusieron a saco. Estava en esta sazón, como dicho es, el Duque de Anjous en la baxa Calabria y pensó que le llamaran para el govierno de la Reyna y púsose en punto, pero como siempre aborrecía al sucessor y al que estava presente, la Duquesa de Sessa, que era muy afizionada al Rey de Aragón, y Juan Cicinello, deseoso de governar todo, no dieron lugar que la Reyna lo mandasse llamar".

Sigue Zurita llamando duque y no rey al d'Anjou; mientras DDM dice que "detta Regina redusse ad executione lo fatto", en Zurita se dice que los conjurados uordenaron a cuatro horas de la noche...... De menos importancia, por tratarse en el capítulo adecuado, es que Zurita traduce que ala gota le subía ya a la cabeça», cuando DDM dice aerali scesa quella gotta da sua testan... Son los imponderables factores aproximativos en la traducción de textos afines, si bien tan puntualmente inobservados en su valor semántico... por traidoras homonimias entre el español e italiano. 
El texto de DDM relativo al rey Luis, que decide ir a Nápoles para gobernar, es significativo: ase lo sconsaro per loro poter bene temporiggiare* los privados de la reina, los conspiradores, entre ellos, la duquesa de Sessa...

2.14. El rey de Aragón, sabido el hecho del asesinato del gran senescal, decide venir a su Ischia desde Sicilia y espera de la duquesa de Sexa su apoyo "che avea il dominio in soa podestá, assai ne fo contento...", pero Urbano Cimmino - ache era gran servitore de Rè Luise che tutto il dì stava ale orecchie sue et dela Regina, la quale come li dicea cosí facea, perché era vecchia et de corta vista, la sconsigliava dello acordo de Ré de Rahona perché la regina ne havea voluntán- logra disuadir a la reina de darle al aragonés las riendas del reino tras renovarle la adopción, que el gran senescal había impedido hasta entonces; Zurita es más preciso en este punto y diverge de DDM y así lo reconoce explícitamente al final del período: Aunque la Duquesa de Sessa era la que tenía en esta sazón absoluto poder sobre la Reyna... y ella desseava que el Rey fuesse llamado y requerido y restituydo en su derecho, la Reyna venía en todo, como no viesse al Rey de sus ojos ni pusiesse mano en lo del govierno, y assí concediò la revocación de la adopción y donación que hizo al Duque de Anjous y confirmó la primera del Rey. Esto fue en gran secreto, porque Urbano Cimino, que estava siempre a las orejas de la Reyna, y otros muchos afizionados al vando Anjoíno no lo entendiessen... porque desta renovación, siendo cosa tan señalada, ninguno de los Autores de aquellos tiempos ni de los que en estos han hecho más diligencia en escrivir las cosas de aquel Reyno, haze mención della, me pareció cosa muy digna de ponerse en este lugar, pues por ella se entiende tanta parte, no sólo de la justificación, pero de la justicia del Rey y de la liviandad de la Reyna y de la malizia de los que la governavan;; aporta Zurita documentación de la nueva adopción, añadiendo que «Por estar en esta razón la Reyna en poder de muchos afizionados al Duque de Anjous y recelándose de algún peligro de su persona, si esto se supiesse, esta escritura se puso en poder de la Duquesa de Sessa, para que lo tuviesse hasta tanto que estuviesse la Reyna en libertad para poder poner en execución lo que avía prometido y jurado y halláronse a esto presentes por parte de la Reyna la Duquesa de Sessa, el Conde Campobasso y Juan Cicinello y por la del Rey Gil Carcirera, Nicolás Especial y Juan de Caltagirón. Ay Autor que escrive que, como el Rey hizo tan gran confiança en el Duque de Sessa, pensando reduzir por su mano muchos barones del Reyno, esto le salió muy al revés de como lo esperava, porque no sólo no huvo ninguno que se moviesse de los que estavan en privança de la Reyna, pero perdió en gran parte la amistad de la Duquesa, según después pareció que, por ser enemiga del Duque, su marido, en un instante se desdeñó quando supo que el Rey seguía aquel camino y que esto fue causa que la Duquesa anduvo muchos días entreteniendo 
al Rey y que se detuviesse en Iscla mucho tiempow. El texto del Autor antiguo en DDM dice: «Et dentro lo mese de febraro anno domini 1433 lo Rè de Rahona accordó lo Duca de sexa credendo che tutti l'altri lo seguissero et non fo neuno altro che havesse prevaricato dela fedelità dela Regina; la Duchessa de Sexa vedendo il Duca suo marito, lo quale havea per gran nemico, havea pigliata la parte de Rè de Rahona fortemente se desdignó et subbito ne venne inimica de Rè de Rahona... Et Rè de Rahona vedendo non poter prevalere in questo et stando ad Ischa schernito et beffato piglio partito concluso et fece tregua per $\mathrm{x}$ anni con la regina et andosi per soi fattin, que Zurita remata a continuación del párrafo apenas citado: "De la tregua que se assentó por el Rey con la Reyna de Nápoles y de su vuelta a Sicilia...., como subraya Zurita en el capítulo siguiente.

2.15. La tregua con la reyna, de febrero de 1433 , iniciada a raíz de la muerte del gran senescal y de la voluntad y efectiva renovación de la adopción por parte de la reina a favor de Alfonso, según documentación de Zurita, apenas indicada, se interrumpe con la muerte de la reina Juana II el 2 de febrero de 1435. El duque d'Anjou había muerto el 15 de noviembre del año anterior.

El Consejo de Nápoles, los 18 de la Bagliva, a los cuatro días de la muerte de la reina deciden manifestar claramente sus bazas, haciendo alzar banderas de la Iglesia, del papa Eugenio, del rey Renato d'Anjou y del reino, indicando y dando señal manifiesta de la estabilidad $y$ firmeza de las alianzas históricas, si bien el mayor feudatario del reino, el príncipe de Tarento, con la ayuda de Juan de Ventimiglia, enviado por el aragonés, va desmembrando la solidez aparente de los angevinos y reforzando el bando de los aragoneses: «tutti soi adherenti e parenti et sequaci: lo Conte de Lo Rito, Messer Cristofaro Gaetano, Rogeri Gaitano, lo Conte Camerlingo fatto per la Regina, Antonello de la Ratta, lo Conte de Livito et multi altri signori, intro questo Joanne de Carramanico (...tradio lo castello e dedelo in mano de lo Conte de Lo Rito...) et tutti questi sopra scritti signori mandaro confortandosi dovesse cacciare in campo ad Gaeta, che tutti loro se ponevano in ordine di venire, vivere et morire con sua Maestà... Ré de Rahona che venne in campo ali 7 di maggio et da giorno in giorno che gionsero per mare et per terra gente, che lo Campo era fatto sí grosso, si ragionava erano dele persone 15 mila...».

Muratori con más claridad dice que el rey Alfonso «....subbito hebbe intelligenza con quello Signori, che non erano al Consiglio di Napolin. Ante tan poderoso despliegue y concentración de fuerzas aragonesas el Consejo de Nápoles manda venir enseguida a Sforza. 